\title{
RELATIONS BETWEEN CLASSES OF POTENTIALLY STABLE SIGN PATTERNS*
}

\author{
ADAM H. BERLINER ${ }^{\dagger}$, D.D. OLESKY ${ }^{\ddagger}$, AND P. VAN DEN DRIESSCHE ${ }^{\S}$
}

\begin{abstract}
Two subsets of the potentially stable sign patterns of order $n$ have recently been defined, namely, those that allow sets of (refined) inertias $\mathbb{S}_{n}$ and $\mathbb{H}_{n}$. For $n=2$ and $n=3$, it is proved that a sign pattern is potentially stable if and only if it is sign stable, allows $\mathbb{S}_{n}$, or allows $\mathbb{H}_{n}$. This result is also true for sign patterns of order 4 with associated graph that is a tree, remains open for non-tree potentially stable sign patterns of order 4 , and is false for potentially stable sign patterns of orders greater than or equal to 5 .
\end{abstract}

Key words. Sign pattern, Potentially stable, Inertia, Refined inertia, Jacobian.

AMS subject classifications. 15B35, 15A18, 05C50.

1. Introduction. The characterization of potentially stable sign patterns of order $n \geq 4$ is a well known unsolved problem dating back at least 50 years (see [15]). Here we consider the relationship of potentially stable sign patterns of order $n$ to sign patterns that allow the set of inertias $\mathbb{S}_{n}$ (see [3]) or the set of refined inertias $\mathbb{H}_{n}$ (see [4]). The sets $\mathbb{S}_{n}$ and $\mathbb{H}_{n}$ are motivated by the onset of instability in the linearization of a dynamical system associated, respectively, with a zero eigenvalue or a pair of pure imaginary eigenvalues. Our results are useful for determining if instability can occur (for example, leading to oscillatory solutions) for a dynamical system when the linearized matrix is known only up to signs (see, e.g., Section 5 of [4]). If the sign pattern allows $\mathbb{H}_{n}$, then oscillatory solutions of the system may arise due to Hopf bifurcation.

To specify the sets $\mathbb{S}_{n}$ and $\mathbb{H}_{n}$, we give the following definitions. A sign pattern of order $n$ is an $n \times n$ matrix $\mathcal{A}_{n}=\left[\alpha_{i j}\right]$ with $\alpha_{i j} \in\{+,-, 0\}$. The sign pattern class of $\mathcal{A}_{n}$, denoted $\mathcal{Q}\left(\mathcal{A}_{n}\right)$, is the set of all $n \times n$ real matrices $A=\left[a_{i j}\right]$ with $\operatorname{sign}\left(a_{i j}\right)=\alpha_{i j}$ for all $i, j$, and a matrix $A \in \mathcal{Q}\left(\mathcal{A}_{n}\right)$ is a realization of $\mathcal{A}$. We only consider sign patterns up to equivalence, i.e., up to permutation similarity, transposition, and/or signature similarity, since these operations leave (refined) inertia invariant. A sign pattern $\mathcal{B}_{n}$ is a superpattern of $\mathcal{A}_{n}$ if $\mathcal{B}_{n}$ is obtained from $\mathcal{A}_{n}$ by replacing some (or none) of the zero entries of $\mathcal{A}_{n}$ by + or - .

The inertia of an $n \times n$ real matrix $A$ is the ordered triple i $(A)=\left(n_{+}, n_{-}, n_{0}\right)$ of nonnegative integers summing to $n$ where $n_{+}$(respectively, $n_{-}, n_{0}$ ) is the number of eigenvalues of $A$ with positive (respectively, negative, zero) real parts. The refined inertia of an $n \times n$ real matrix $A$ is the ordered 4 -tuple $\operatorname{ri}(A)=$ $\left(n_{+}, n_{-}, n_{z}, 2 n_{p}\right)$, where $n_{z}$ is the number of zero eigenvalues and $2 n_{p}$ is the number of nonzero pure imaginary eigenvalues of $A$ [13]. Note that $n_{0}=n_{z}+2 n_{p}$.

The inertia of a sign pattern $\mathcal{A}_{n}$ is $\mathrm{i}\left(\mathcal{A}_{n}\right)=\left\{\mathrm{i}(A): A \in \mathcal{Q}\left(\mathcal{A}_{n}\right)\right\}$. Similarly, the refined inertia of a sign pattern $\mathcal{A}_{n}$ is $\operatorname{ri}\left(\mathcal{A}_{n}\right)=\left\{\operatorname{ri}(A): A \in \mathcal{Q}\left(\mathcal{A}_{n}\right)\right\}$. If $\mathcal{P}$ is any subset of inertias (respectively, refined inertias), then $\mathcal{A}_{n}$ allows $\mathcal{P}$ if $\mathcal{P} \subseteq \mathrm{i}\left(\mathcal{A}_{n}\right)$ (respectively, $\mathcal{P} \subseteq \operatorname{ri}\left(\mathcal{A}_{n}\right)$ ) and $\mathcal{A}_{n}$ requires $\mathcal{P}$ if $\mathcal{P}=\mathrm{i}\left(\mathcal{A}_{n}\right)$ (respectively, $\left.\mathcal{P}=\operatorname{ri}\left(\mathcal{A}_{n}\right)\right)$. Sign pattern $\mathcal{A}_{n}$ is sign stable if $\mathrm{i}\left(\mathcal{A}_{n}\right)=\{(0, n, 0)\}$, potentially stable if $(0, n, 0) \in \mathrm{i}\left(\mathcal{A}_{n}\right)$,

* Received by the editors on August 24, 2019. Accepted for publication on February 28, 2020. Handling Editor: Froilán M. Dopico. Corresponding Author: Adam H. Berliner.

${ }^{\dagger}$ MSCS Department, St. Olaf College, Northfield, Minnesota 55057, USA (berliner@stolaf.edu).

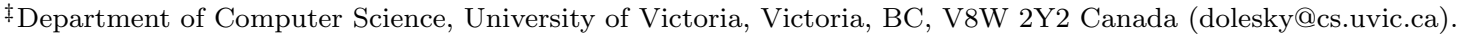

$\S$ Department of Mathematics and Statistics, University of Victoria, Victoria, BC, V8W 2Y2 Canada (pvdd@math.uvic.ca). 
and minimally potentially stable if it is irreducible and potentially stable with the property that changing any nonzero sign to 0 results in a sign pattern that is not potentially stable. Superpatterns of potentially stable sign patterns and direct sums of potentially stable sign patterns are also potentially stable. Thus, only irreducible potentially stable sign patterns need to be considered.

We focus on potentially stable irreducible sign patterns that allow or require the set of inertias

$$
\mathbb{S}_{n}=\{(0, n, 0),(0, n-1,1),(1, n-1,0)\} \quad(n \geq 2)
$$

or the set of refined inertias

$$
\mathbb{H}_{n}=\{(0, n, 0,0),(0, n-2,0,2),(2, n-2,0,0)\} \quad(n \geq 3) .
$$

Thus, $\mathcal{A}_{n}$ allows $\mathbb{S}_{n}$ if $\mathbb{S}_{n} \subseteq \mathrm{i}\left(\mathcal{A}_{n}\right)$ and requires $\mathbb{S}_{n}$ if $\mathbb{S}_{n}=\mathrm{i}\left(\mathcal{A}_{n}\right)$, with a similar statement for $\mathbb{H}_{n}$. Obviously if $\mathcal{A}_{n}$ requires $\mathbb{S}_{n}\left(\mathbb{H}_{n}\right)$, then $\mathcal{A}_{n}$ allows $\mathbb{S}_{n}\left(\mathbb{H}_{n}\right)$. If $\mathcal{A}_{n}$ is sign stable, allows $\mathbb{S}_{n}$, or allows $\mathbb{H}_{n}$, then clearly $\mathcal{A}_{n}$ is potentially stable.

The sign pattern $\mathcal{A}_{n}=\left[\alpha_{i j}\right]$ is combinatorially symmetric when $\alpha_{i j} \neq 0$ if and only if $\alpha_{j i} \neq 0$. If $\mathcal{A}_{n}$ is combinatorially symmetric and irreducible, then $\mathcal{A}_{n}$ is associated with an undirected, connected, signed graph $G$. The graph $G$ has vertex set $\{1,2, \ldots, n\}$, a loop at vertex $i$ signed as $\alpha_{i i}$ if and only if $\alpha_{i i} \neq 0$, and an edge between vertices $i$ and $j$ signed as $\alpha_{i j} \alpha_{j i}$ if and only if $\alpha_{i j} \neq 0$. We primarily focus on tree sign patterns, i.e., patterns associated with connected graphs having no cycles of length 3 or longer. The graph of a star sign pattern is a tree with one central vertex connected by an edge to all other vertices, while the graph of a path sign pattern is a tree where each vertex is connected by an edge to no more than two other vertices. For $n=3$, every tree sign pattern is equivalent to a path sign pattern; and for $n=4$, every tree sign pattern is equivalent to a star or a path sign pattern.

2. Preliminary results. We present some results from the recent literature that relate to the sets $\mathbb{S}_{n}$ and $\mathbb{H}_{n}$.

The Jacobian matrix associated with a sign pattern plays an important role in studying the sets $\mathbb{S}_{n}$ and $\mathbb{H}_{n}$ (see, e.g., $[2,3]$ ). Let $A=\left[a_{i j}\right]$ be a (real) matrix of order $n$ with $m \geq n$ nonzero entries. Form matrix $X$ by replacing the $m$ nonzero entries $a_{i_{1} j_{1}}, \ldots, a_{i_{m} j_{m}}$ of $A$ by variables $x_{1}, \ldots, x_{m}$. The characteristic polynomial of $X$ is

$$
z^{n}+p_{1} z^{n-1}+\cdots+p_{n-1} z+p_{n}
$$

where $p_{1}, \ldots, p_{n}$ depend on $x_{1}, \ldots, x_{m}$. Compute the Jacobian matrix $J$ with $(i, j)$-entry $\frac{\partial p_{i}\left(x_{1}, \ldots, x_{m}\right)}{\partial x_{j}}$ evaluated at $x_{k}=a_{i_{k} j_{k}}$ for $1 \leq k \leq m$. If $J$ has rank $n$, then $A$ allows a Jacobian of full rank. This Jacobian matrix and the implicit function theorem are used to prove the results in the following theorem.

Theorem 1. ([3, Theorem 2.2], [2, Theorem 3.1]) Let $\mathcal{A}_{n}$ be a sign pattern of order $n$ and $A \in \mathcal{Q}\left(\mathcal{A}_{n}\right)$. If $\mathrm{i}(A)=(0, n-1,1)$ or $\operatorname{ri}(A)=(0, n-2,0,2)$ and $A$ allows a Jacobian of full rank, then $\mathcal{A}_{n}$ and any superpattern of $\mathcal{A}_{n}$ allow $\mathbb{S}_{n}$ or $\mathbb{H}_{n}$, respectively.

Using a Jacobian matrix of full rank, a useful method for constructing sign patterns of order $n+1$ that allow $\mathbb{S}_{n+1}$ from a sign pattern of order $n$ that allows $\mathbb{S}_{n}$ is given in [1]. We extend this result so that it also applies to $\mathbb{H}_{n}$, with a similar proof (where $\left(n_{+}, n_{-}, n_{0}\right)$ is replaced by $\left(n_{+}, n_{-}, n_{z}, 2 n_{p}\right)$ and $(0, n-1,1)$ is replaced by $(0, n-2,0,2))$. 
Theorem 2. ([1, Theorem 3.1 and Corollary 3.2]) Let $\mathcal{A}_{n}$ be a sign pattern of order $n$ that has at least $n$ nonzero entries and define $\mathcal{A}_{n+1}=\mathcal{A}_{n} \oplus[-]$. If $\mathcal{A}_{n}$ allows $\mathbb{S}_{n}$ (respectively, $\mathbb{H}_{n}$ ) and has a realization $A$ with $\mathrm{i}(A)=(0, n-1,1)$ (respectively, $\operatorname{ri}(A)=(0, n-2,0,2))$ that allows a Jacobian of full rank, then every superpattern of $\mathcal{A}_{n+1}$ allows $\mathbb{S}_{n+1}$ (respectively, $\mathbb{H}_{n+1}$ ), and also has a realization $B$ with $\mathrm{i}(B)=(0, n, 1)$ (respectively, $\operatorname{ri}(B)=(0, n-1,0,2))$ that allows a Jacobian of full rank.

3. Potentially stable sign patterns of order $n \leq 3$. We begin our study of relationships between potentially stable sign patterns and certain subsets of them by considering orders 2 and 3 .

THEOREM 3. An irreducible sign pattern of order 2 is potentially stable if and only if it is sign stable or allows $\mathbb{S}_{2}$.

Proof. A potentially stable sign pattern must have at least one negative diagonal entry, and for order 2 must allow a positive determinant. Thus, up to equivalence, there are four irreducible potentially stable sign patterns of order 2 :

$$
\mathcal{B}_{2,1}=\left[\begin{array}{cc}
- & + \\
- & 0
\end{array}\right], \quad \mathcal{B}_{2,2}=\left[\begin{array}{ll}
- & + \\
- & -
\end{array}\right], \quad \mathcal{B}_{2,3}=\left[\begin{array}{ll}
- & + \\
- & +
\end{array}\right], \quad \mathcal{B}_{2,4}=\left[\begin{array}{ll}
- & + \\
+ & -
\end{array}\right]
$$

Patterns $\mathcal{B}_{2,1}$ and $\mathcal{B}_{2,2}$ are sign stable, $\mathcal{B}_{2,3}$ allows $\mathbb{S}_{2}$, and $\mathcal{B}_{2,4}$ requires $\mathbb{S}_{2}$. The converse is clearly true.

THEOREM 4. A minimally potentially stable sign pattern of order 3 is either sign stable, requires $\mathbb{S}_{3}$, or requires $\mathbb{H}_{3}$. Moreover, if a minimally potentially stable sign pattern of order 3 requires $\mathbb{S}_{3}$ (respectively, $\mathbb{H}_{3}$ ), then every superpattern allows $\mathbb{S}_{3}$ (respectively, $\mathbb{H}_{3}$ ).

Proof. Up to equivalence, there are five minimally potentially stable sign patterns of order 3 (see, e.g., [16] and page 53 of [18]):

$$
\begin{gathered}
\mathcal{C}_{3,1}=\left[\begin{array}{ccc}
- & + & 0 \\
- & 0 & + \\
0 & - & 0
\end{array}\right], \quad \mathcal{C}_{3,2}=\left[\begin{array}{ccc}
+ & + & 0 \\
- & - & + \\
0 & + & 0
\end{array}\right], \quad \mathcal{C}_{3,3}=\left[\begin{array}{ccc}
- & + & 0 \\
0 & - & + \\
- & 0 & 0
\end{array}\right], \\
\mathcal{C}_{3,4}=\left[\begin{array}{ccc}
- & + & 0 \\
- & 0 & + \\
- & 0 & 0
\end{array}\right], \quad \mathcal{C}_{3,5}=\left[\begin{array}{ccc}
- & + & 0 \\
0 & 0 & + \\
+ & - & 0
\end{array}\right] .
\end{gathered}
$$

Pattern $\mathcal{C}_{3,1}$ is sign stable, whereas $\mathcal{C}_{3,2}, \mathcal{C}_{3,3}, \mathcal{C}_{3,4}$ require $\mathbb{H}_{3}$ (see Section 3 of [10]) and $\mathcal{C}_{3,5}$ requires $\mathbb{S}_{3}$ (see Example 1.1 of [3]). The following realizations of $\mathcal{C}_{3,2}, \mathcal{C}_{3,3}$, and $\mathcal{C}_{3,4}$ (respectively) achieve refined inertia $(0,1,0,2)$ :

$$
C_{3,2}=\left[\begin{array}{ccc}
1 & 1 & 0 \\
-4 & -2 & 1 \\
0 & 1 & 0
\end{array}\right], \quad C_{3,3}=\left[\begin{array}{ccc}
-1 & 1 & 0 \\
0 & -2 & 1 \\
-6 & 0 & 0
\end{array}\right], \quad C_{3,4}=\left[\begin{array}{ccc}
-1 & 1 & 0 \\
-1 & 0 & 1 \\
-1 & 0 & 0
\end{array}\right]
$$

Each of these realizations allows a Jacobian of full rank, and thus, every superpattern of $\mathcal{C}_{3,2}, \mathcal{C}_{3,3}, \mathcal{C}_{3,4}$ allows $\mathbb{H}_{3}$ by Theorem 1. The following realization of $\mathcal{C}_{3,5}$ achieves inertia $(0,2,1)$ :

$$
C_{3,5}=\left[\begin{array}{ccc}
-1 & 1 & 0 \\
0 & 0 & 1 \\
1 & -1 & 0
\end{array}\right]
$$

Since $C_{3,5}$ allows a Jacobian of full rank, the superpatterns of $\mathcal{C}_{3,5}$ allow $\mathbb{S}_{3}$.

TheOREM 5. A tree (path) sign pattern of order 3 is potentially stable if and only if it is sign stable, allows $\mathbb{S}_{3}$, or allows $\mathbb{H}_{3}$. Moreover, if a tree sign pattern of order 3 allows $\mathbb{S}_{3}$ (respectively, $\mathbb{H}_{3}$ ), then every superpattern allows $\mathbb{S}_{3}$ (respectively, $\mathbb{H}_{3}$ ). 
Proof. Clearly, a sign pattern that is sign stable or allows $\mathbb{S}_{3}$ or $\mathbb{H}_{3}$ is potentially stable. Without loss of generality, a path sign pattern of order 3 can be specified as $\left[\begin{array}{ccc}a_{1} & + & 0 \\ b_{2} & a_{2} & + \\ 0 & b_{3} & a_{3}\end{array}\right]$ with $b_{i} \neq 0$. We denote such a pattern by the sequence of signs $a_{1} a_{2} a_{3} / b_{2} b_{3}$.

The 18 potentially stable path sign patterns of order 3 (up to equivalence) are listed in Figure 2 of [12]. Four of these sign patterns are sign stable, namely $\ominus \ominus-/--$, where $\ominus$ denotes 0 or - . We then consider the following six sign patterns:

\begin{tabular}{|c|c|c|}
\hline Path sign pattern & Result & Realization(s) \\
\hline$+-0 /-+$ & requires $\mathbb{H}_{3}$ (see sign pattern $\mathcal{C}_{3,2}$ above) & $1,-2,0 /-4,1$ \\
\hline $0+-/--$ & requires $\mathbb{H}_{3}$ (see 3.1 in $\left.[10]\right)$ & $0,1,-2 /-2,-4$ \\
\hline$+0-/--$ & allows $\mathbb{S}_{3}$ and $\mathbb{H}_{3}$ (see Appendix $\mathrm{B}(12 \mathrm{a})$ in [17]) & $1,0,-2 /-1,-2$ \\
\hline$-0-/+-$ & allows $\mathbb{S}_{3}$ and $\mathbb{H}_{3}$ (see Appendix $\mathrm{B}(11 \mathrm{a})$ in $\left.[17]\right)$ ) & $\begin{array}{l}1,0,-4 /-4,-4 \\
-1,0,-2 / 1,-2\end{array}$ \\
\hline $0--/-+$ & requires $\mathbb{H}_{3}$ (see 3.1 in $\left.[10]\right)$ & $\begin{array}{l}-2,0,-1 / 5,-4 \\
0,-1,-1 /-2,2\end{array}$ \\
\hline$---/++$ & requires $\mathbb{S}_{3}$ (see Appendix $\mathrm{B}(10 \mathrm{a})$ in $\left.[17]\right)$ ) & $-1,-2,-1 / 1,1$ \\
\hline
\end{tabular}

For the six sign patterns above that require or allow $\mathbb{S}_{3}$ or $\mathbb{H}_{3}$, the realizations given have every superdiagonal entry equal to 1 and inertia $(0,2,1)$ or refined inertia $(0,1,0,2)$, respectively, that allow a Jacobian of full rank. The remaining eight potentially stable path sign patterns in [12] are superpatterns of these six, and thus allow $\mathbb{S}_{3}$ or $\mathbb{H}_{3}$ by Theorem 1 , and the first result follows. The second result also follows from Theorem 1.

There are other potentially stable (non-tree) sign patterns of order 3 that are not included in Theorems 4 or 5 . For example, $\left[\begin{array}{ccc}- & + & 0 \\ 0 & - & + \\ + & 0 & -\end{array}\right]$ requires $\mathbb{S}_{3}$ and the realization $\left[\begin{array}{ccc}-1 & 1 & 0 \\ 0 & -2 & 1 \\ 2 & 0 & -1\end{array}\right]$ has inertia $(0,2,1)$ and allows a Jacobian of full rank; thus, every superpattern of this sign pattern allows $\mathbb{S}_{3}$.

THEOREM 6. An irreducible sign pattern of order 3 is potentially stable if and only if it is sign stable, allows $\mathbb{S}_{3}$, or allows $\mathbb{H}_{3}$.

Proof. Since the converse is clear, assume that $\mathcal{A}_{3}$ is a potentially stable irreducible sign pattern of order 3. The result is true for minimally potentially stable sign patterns (Theorem 4), tree sign patterns (Theorem $5)$, as well as all of their superpatterns.

Berliner et al. (see Figure 2 and Appendices A and B of [1]) give all 200 nonequivalent sign patterns of order 3 for which any realization has at least two nonzero terms in its determinant expansion. We have determined that each of these sign patterns is a superpattern of a sign pattern previously identified in this section as being sign stable, allowing $\mathbb{S}_{3}$, or allowing $\mathbb{H}_{3}$, or either is not potentially stable as checked by the Routh-Hurwitz criteria (see, e.g., [4]).

We are left to consider irreducible potentially stable non-tree sign patterns $\mathcal{A}_{3}$ with exactly one nonzero term in the determinant expansion of any realization. Thus, without loss of generality, for any $A \in \mathcal{Q}\left(\mathcal{A}_{3}\right)$, this nonzero term is $a_{12} a_{23} a_{31}$. Since $\mathcal{A}_{3}$ is potentially stable, $a_{12} a_{23} a_{31}$ must be negative. If $a_{i j} a_{j i}=0$ for all $i, j$ where $i \neq j$, then $A$ must have exactly two negative diagonal entries in order to achieve stability, giving a sign pattern equivalent to $\mathcal{C}_{3,3}$. If $a_{i j} a_{j i} \neq 0$ for some $i, j$ where $i \neq j$, we may assume without loss of generality that $a_{12} a_{21} \neq 0$. If $a_{12} a_{21}<0$, then in addition, at least one diagonal entry must be negative, 
and the two possibilities are $a_{11}$ or $a_{22}$. These choices are equivalent, and the overall result is equivalent to a superpattern of $\mathcal{C}_{3,4}$. On the other hand, if $a_{12} a_{21}>0$, then $a_{33}=0$. Then either both or one of $a_{11}$ and $a_{22}$ are negative. In the first case, $\mathcal{A}_{3}$ is equivalent to a superpattern of $\mathcal{C}_{3,3}$. If only $a_{11}<0$, then $a_{13}>0$ and $\mathcal{A}_{3}$ is equivalent to a superpattern of $\mathcal{C}_{3,4}$. If only $a_{22}<0$, then $a_{32}<0$ and $\mathcal{A}_{3}$ is equivalent to a superpattern of $\mathcal{C}_{3,4}$. From Theorem 4 , both $\mathcal{C}_{3,3}$ and $\mathcal{C}_{3,4}$ and their superpatterns allow $\mathbb{H}_{3}$.

4. Potentially stable sign patterns of order 4 . If $\mathcal{A}$ is a tree sign pattern of order 4 , then its associated graph is either a star or a path. We consider each case separately below. For these sign patterns, we have determined exactly those that allow $\mathbb{S}_{4}$ and/or $\mathbb{H}_{4}$ or require $\mathbb{S}_{4}$ or $\mathbb{H}_{4}$. Note that Gao et al. ([5, 6]) have classified all star sign patterns that require $\mathbb{H}_{n}$.

THEOREM 7. A star sign pattern of order 4 is potentially stable if and only if it is sign stable, allows $\mathbb{S}_{4}$, or allows $\mathbb{H}_{4}$. Moreover, if a star sign pattern of order 4 allows $\mathbb{S}_{4}$ (respectively, $\mathbb{H}_{4}$ ), then every superpattern allows $\mathbb{S}_{4}$ (respectively, $\mathbb{H}_{4}$ ).

Proof. Without loss of generality, a star sign pattern of order 4 can be specified as $\left[\begin{array}{cccc}a_{1} & + & + & + \\ b_{2} & a_{2} & 0 & 0 \\ b_{3} & 0 & a_{3} & 0 \\ b_{4} & 0 & 0 & a_{4}\end{array}\right]$ with $b_{i} \neq 0$. We denote such a pattern by the sequence of signs $a_{1} a_{2} a_{3} a_{4} / b_{2} b_{3} b_{4}$. Note that rows and columns $2,3,4$ can be simultaneously permuted to give an equivalent sign pattern.

Since the converse is clear, assume that the sign pattern is potentially stable. A full list of potentially stable star sign patterns of order 4 is given in Table 1 of [12]. Four of these patterns are sign stable, namely $\ominus \ominus--/---$. Ten more sign patterns that allow or require $\mathbb{S}_{4}$ or $\mathbb{H}_{4}$ are given below. Either Theorem 2 is used with a sign pattern of order 3 that is equivalent to one of the path sign patterns in the proof of Theorem 5 , or there is a star sign pattern realization given with every off-diagonal entry in the first row equal to 1 and inertia $(0,3,1)$ or refined inertia $(0,2,0,2)$, respectively, that allows a Jacobian of full rank.

\begin{tabular}{c|l|l} 
Star sign pattern & \multicolumn{1}{|c}{ Result } & \multicolumn{1}{|c}{ Reason/Realization } \\
\hline$----/+++$ & requires $\mathbb{S}_{4}\left(\right.$ see $\mathcal{S}_{2}$ in $\left.[9]\right)$ & Theorem 12 in Section 5 \\
$---0 /++-$ & requires $\mathbb{H}_{4}($ Theorem 2 with path $0--/-+$ \\
$--+0 /--+$ & allows $\mathbb{H}_{4}\left(\right.$ see $\mathcal{S}_{6}$ in $\left.[9]\right)$ & Theorem 2 with path $+-0 /-+$ \\
$--+0 /+-+$ & requires $\mathbb{H}_{4}\left(\right.$ see $\mathcal{S}_{4}$ in $\left.[9]\right)$ & Theorem 2 with path $+-0 /-+$ \\
$-++0 /+--$ & allows $\mathbb{H}_{4}\left(\right.$ see $\mathcal{S}_{7}$ in $\left.[9]\right)$ & $-1,0.125,0.25,0 / 8.125,-8.5,-1$ \\
$0---/-++$ & allows $\mathbb{S}_{4}$ and $\mathbb{H}_{4}$ & Theorem 2 with path $-0-/+-$ \\
$00--/-+-$ & requires $\mathbb{H}_{4}\left(\right.$ see $\mathcal{S}_{3}$ in $\left.[9]\right)$ & $0,0,-1,-1 /-1,1,-1$ \\
$0+--/---$ & allows $\mathbb{S}_{4}$ and $\mathbb{H}_{4}$ & Theorem 2 with path $+0-/--$ \\
$00+-/+--$ & allows $\mathbb{H}_{4}\left(\right.$ see $\mathcal{S}_{8}$ in $\left.[9]\right)$ & $0,0,0.5,-1 / 1.5,-2,-2$ \\
$+0--/---$ & requires $\mathbb{H}_{4}\left(\right.$ see $\mathcal{S}_{5}$ in $\left.[9]\right)$ & Theorem 2 with path $0+-/--$
\end{tabular}

The remaining potentially stable star sign patterns in [12] are superpatterns of one of the ten sign patterns above. Thus, by Theorem 1, each sign pattern and their superpatterns allow $\mathbb{S}_{4}$ or $\mathbb{H}_{4}$.

THEOREM 8. A path sign pattern of order 4 is potentially stable if and only if it is sign stable, allows $\mathbb{S}_{4}$, or allows $\mathbb{H}_{4}$. Moreover, if a path sign pattern of order 4 allows $\mathbb{S}_{4}$ (respectively, $\mathbb{H}_{4}$ ), then every superpattern allows $\mathbb{S}_{4}$ (respectively, $\mathbb{H}_{4}$ ). 
Proof. Without loss of generality, a path sign pattern of order 4 can be specified as $\left[\begin{array}{cccc}a_{1} & + & 0 & 0 \\ b_{2} & a_{2} & + & 0 \\ 0 & b_{3} & a_{3} & + \\ 0 & 0 & b_{4} & a_{4}\end{array}\right]$ with $b_{i} \neq 0$. We denote such a pattern by the sequence of signs $a_{1} a_{2} a_{3} a_{4} / b_{2} b_{3} b_{4}$. Note that rows and columns 1,4 and rows and columns 2, 3 can be simultaneously interchanged to give an equivalent sign pattern.

Since the converse is clear, assume that the sign pattern is potentially stable. Order 4 potentially stable path sign patterns were specified in [12], and this list was completed with four additional patterns $\mathcal{A}_{4,8}, \mathcal{A}_{4,9}, \mathcal{A}_{4,10}, \mathcal{A}_{4,11}$ in [14]. We start with the sign patterns given by $\ominus \ominus \ominus-/---$ and $0-\ominus 0 /---$, which are sign stable. The remaining potentially stable path sign patterns are either in the following list or are superpatterns of these. If a numerical realization is given, it has each superdiagonal entry equal to 1 , achieves inertia $(0,3,1)$ or refined inertia $(0,2,0,2)$, and allows a Jacobian of full rank. Otherwise, Theorem 2 is used with a sign pattern of order 3 that is equivalent to one of the path sign patterns in the proof of Theorem 5 .

\begin{tabular}{|c|c|c|}
\hline Path sign pattern & Result & Reason/Realization \\
\hline $00+-/---$ & allows $\mathbb{H}_{4}$ & $0,0,0.5,-1 /-0.5,-0.25,-1$ \\
\hline$+00-/---$ & allows $\mathbb{S}_{4}$ and allows $\mathbb{H}_{4}$ & $\begin{array}{l}1,0,0,-2 /-2,-3,-3 \\
1,0,0,-2 /-1.5,-1,-3\end{array}$ \\
\hline $0+0-/---$ & allows $\mathbb{H}_{4}\left(\right.$ see $\mathcal{P}_{6}$ in [9]) & $0,1,0,-2 /-0.25,-1.25,-2$ \\
\hline$+-0-/-++$ & allows $\mathbb{S}_{4}$ and allows $\mathbb{H}_{4}$ & $\begin{array}{l}1,-2,0,-2 /-3,0.5,1 \\
\text { Theorem } 2 \text { with path }+-0 /-+\end{array}$ \\
\hline$-00-/+--$ & allows $\mathbb{S}_{4}$ and allows $\mathbb{H}_{4}$ & $\begin{array}{l}-1,0,0,-2 / 1,-1,-2 \\
-1,0,0,-2 / 2,-2,-1\end{array}$ \\
\hline$-00-/-+-$ & allows $\mathbb{S}_{4}$ and allows $\mathbb{H}_{4}$ & $\begin{array}{l}-4,0,0,-0.5 /-1,0.5,-1 \\
-1,0,0,-1 /-1.5,1.25,-3\end{array}$ \\
\hline $0-0-/-+-$ & requires $\mathbb{H}_{4}\left(\right.$ see $\mathcal{P}_{1}$ in $\left.[7,9]\right)$ & $0,-1,0,-2 /-3,2,-1$ \\
\hline $0-0-/+-+$ & requires $\mathbb{H}_{4}\left(\right.$ see $\mathcal{P}_{2}$ in $\left.[7,9]\right)$ & $0,-1,0,-1 / 2,-5,1$ \\
\hline $00--/--+$ & allows $\mathbb{S}_{4}$ & $0,0,-1,-1 /-1,-1,1$ \\
\hline $00--/+-+$ & allows $\mathbb{S}_{4}$ and allows $\mathbb{H}_{4}$ & $\begin{array}{l}0,0,-1,-2 / 1,-2,2 \\
0,0,-1,-1 / 1,-4,2\end{array}$ \\
\hline$-0--/-++$ & allows $\mathbb{S}_{4}$ and $\mathbb{H}_{4}$ & Theorem 2 with path $-0-/+-$ \\
\hline $0---/-++$ & allows $\mathbb{S}_{4}$ and allows $\mathbb{H}_{4}$ & $\begin{array}{l}0,-1,-1,-1 /-1,1,1 \\
\text { Theorem } 2 \text { with path } 0--/-+\end{array}$ \\
\hline$----/+++$ & allows $\mathbb{S}_{4}$ & Theorem 2 with path $---/++$ \\
\hline $00-+/---$ & allows $\mathbb{S}_{4}$ and allows $\mathbb{H}_{4}$ & $\begin{array}{l}0,0,-1,0.5 /-2,-1,-0.5 \\
0,0,-1,0.5 /-2,-1,-2.5\end{array}$ \\
\hline $00-+/-+-$ & allows $\mathbb{H}_{4}$ & $0,0,-1,0.5 /-0.5,0.5,-2.5$ \\
\hline$+0-0 /---$ & allows $\mathbb{H}_{4}\left(\right.$ see $\mathcal{P}_{8}$ in [9]) & $1,0,-2,0 /-2,-2,-1$ \\
\hline $0+-0 /---$ & allows $\mathbb{H}_{4}\left(\right.$ see $\mathcal{P}_{10}$ in [9]) & $0,1,-2,0 /-1.5,-2.5,-2$ \\
\hline$-+-0 /--+$ & allows $\mathbb{S}_{4}$ and allows $\mathbb{H}_{4}$ & $\begin{array}{l}-1,1,-3,0 /-1,-2,1 \\
\text { Theorem } 2 \text { with path }+-0 /-+\end{array}$ \\
\hline $0--0 /-+-$ & requires $\mathbb{H}_{4}\left(\right.$ see $\mathcal{P}_{4}$ in $\left.[7,9]\right)$ & $0,-1,-2,0 /-1,2,-1$ \\
\hline $00+-/-+-$ & allows $\mathbb{S}_{4}$ and allows $\mathbb{H}_{4}$ & $\begin{array}{l}0,0,1,-3 /-2,1,-3 \\
0,0,0.5,-1 /-2,0.5,-1\end{array}$ \\
\hline$+00-/+-+$ & allows $\mathbb{S}_{4}$ and allows $\mathbb{H}_{4}$ & $\begin{array}{l}1,0,0,-1.6 / 6.4,-12.8,3.2 \\
1,0,0,-2 / 9,-\frac{202}{13}, \frac{45}{13}\end{array}$ \\
\hline $0+-0 /+-+$ & allows $\mathbb{H}_{4}\left(\right.$ see $\mathcal{P}_{11}$ in [9]) & $0,1,-2,0 / 1,-10,3$ \\
\hline $0--+/++-$ & allows $\mathbb{S}_{4}$ and allows $\mathbb{H}_{4}$ & $\begin{array}{l}0,-1,-2,1 / 0.25,0.5,-2 \\
0,-1,-9,2 / \frac{1}{11}, \frac{53}{11},-17\end{array}$ \\
\hline
\end{tabular}

By Theorem 1, each sign pattern above and their superpatterns allow $\mathbb{S}_{4}$ or $\mathbb{H}_{4}$. 
Note that the three path sign patterns that require $\mathbb{H}_{4}$ are given in [7]; they list exactly five sign patterns that require $\mathbb{H}_{4}$, but two are superpatterns of others. Combining Theorems 7 and 8 , we obtain the following result.

TheOREm 9. A tree sign pattern of order 4 is potentially stable if and only if it is sign stable, allows $\mathbb{S}_{4}$, or allows $\mathbb{H}_{4}$. Moreover, if a tree sign pattern of order 4 allows $\mathbb{S}_{4}$ (respectively, $\mathbb{H}_{4}$ ), then every superpattern allows $\mathbb{S}_{4}$ (respectively, $\mathbb{H}_{4}$ ).

5. Potentially stable sign patterns of arbitrary order. Whereas the previous sections focused on sign patterns of order $n \leq 4$, here some families of potentially stable sign patterns of arbitrary order are considered, and it is shown that these sign patterns allow either $\mathbb{S}_{n}$ or $\mathbb{H}_{n}$. We also give a family of sign patterns that require $\mathbb{S}_{n}$ for $n \geq 2$.

ExAmPLE 10. Consider the star sign pattern $\mathcal{X}_{4}$ and the realization $X_{4}$ given by

$$
\mathcal{X}_{4}=\left[\begin{array}{cccc}
0 & + & + & + \\
+ & 0 & 0 & 0 \\
- & 0 & + & 0 \\
- & 0 & 0 & -
\end{array}\right] \quad \text { and } \quad X_{4}=\left[\begin{array}{cccc}
0 & 1 & 1 & 1 \\
1.5 & 0 & 0 & 0 \\
-2 & 0 & 0.5 & 0 \\
-2 & 0 & 0 & -1
\end{array}\right]
$$

Sign pattern $\mathcal{X}_{4}$ is potentially stable (denoted by $00+-/+--$ in the proof of Theorem 7 ) and allows $\mathbb{H}_{4}$. The realization $X_{4}$ allows a Jacobian of full rank for which $\operatorname{ri}\left(X_{4}\right)=(0,2,0,2)$, and thus, by Theorem 2 , there is a realization $X_{5}$ of

$$
\mathcal{X}_{5}=\left[\begin{array}{ccccc}
0 & + & + & + & + \\
+ & 0 & 0 & 0 & 0 \\
- & 0 & + & 0 & 0 \\
- & 0 & 0 & - & 0 \\
\pm & 0 & 0 & 0 & -
\end{array}\right]
$$

allowing a Jacobian of full rank for which $\operatorname{ri}\left(X_{5}\right)=(0,3,0,2)$. Thus, $\mathcal{X}_{5}$ allows $\mathbb{H}_{5}$.

Theorem 2 can be repeatedly applied to $\mathcal{X}_{5}$ (and all subsequent derived sign patterns) in the same way, to obtain a family of sign patterns of all orders $n \geq 4$,

$$
\mathcal{X}_{n}=\left[\begin{array}{cccccccc}
0 & + & + & + & + & \cdots & + & + \\
+ & 0 & 0 & 0 & 0 & \cdots & 0 & 0 \\
- & 0 & + & 0 & 0 & \cdots & 0 & 0 \\
- & 0 & 0 & - & 0 & \cdots & 0 & 0 \\
\pm & 0 & 0 & 0 & - & \ddots & \vdots & \vdots \\
\vdots & \vdots & & & & \ddots & \ddots & \vdots \\
\pm & 0 & 0 & 0 & 0 & \cdots & - & 0 \\
\pm & 0 & 0 & 0 & 0 & \cdots & 0 & -
\end{array}\right] .
$$

Note that the potential stability of $\mathcal{X}_{n}$ follows from Theorem 4.2 in [8]. By Theorem 2, each $\mathcal{X}_{n}$ has a realization $X_{n}$ that allows a Jacobian of full rank for which $\operatorname{ri}\left(X_{n}\right)=(0, n-2,0,2)$, and so $\mathcal{X}_{n}$ allows $\mathbb{H}_{n}$. Furthermore, by Theorem 1 , every superpattern of $\mathcal{X}_{n}$ also allows $\mathbb{H}_{n}$. Note that $\mathcal{X}_{n}$ does not require $\mathbb{H}_{n}$ by Theorem 1.1 in [6] and does not allow $\mathbb{S}_{n}$ since the determinant of any realization is nonzero.

EXAMPLE 11. The potentially stable sign pattern

$$
\mathcal{Y}_{3}=\left[\begin{array}{ccc}
- & + & 0 \\
0 & - & + \\
+ & 0 & -
\end{array}\right]
$$


requires $\mathbb{S}_{3}$ (see Section 3). More generally, for $n \geq 3$, each sign pattern

$$
\mathcal{Y}_{n}=\left[\begin{array}{cccccc}
- & + & 0 & 0 & \cdots & 0 \\
0 & - & + & 0 & \cdots & 0 \\
\vdots & & \ddots & \ddots & \ddots & \vdots \\
\vdots & & & \ddots & \ddots & \vdots \\
0 & 0 & \ldots & \cdots & - & + \\
+ & 0 & \ldots & \cdots & 0 & -
\end{array}\right]
$$

is potentially stable. The inertia of the realization $Y_{n}$ of $\mathcal{Y}_{n}$ with all nonzero entries having magnitude 1 is $(0, n-1,1)$, since the eigenvalues of $Y_{n}$ are $\left\{\lambda_{i}-1 \mid \lambda_{i}^{n}=1\right\}$. The remaining two inertias in $\mathbb{S}_{n}$ are obtained by realizations $Y_{n} \pm \varepsilon I_{n}$ (for sufficiently small $\varepsilon$ ). Thus, $\mathcal{Y}_{n}$ allows $\mathbb{S}_{n}$. In fact, it can be shown that $\mathcal{Y}_{4}$ requires $\mathbb{S}_{4}$. However $\mathcal{Y}_{n}$ does not require $\mathbb{S}_{n}$ for $n \geq 5$, since the realization of $\mathcal{Y}_{n}$ with all positive entries equal to 1 and diagonal entries sufficiently small in magnitude has at least three eigenvalues with positive real parts. Note that $\mathcal{Y}_{n}$ does not allow $\mathbb{H}_{n}$ since $\mathcal{Y}_{n}$ is irreducible with all off-diagonal entries nonnegative, implying that (by Perron-Frobenius) the eigenvalue with maximum real part of any realization is real and simple, thus precluding the refined inertia $(0, n-2,0,2)$.

THEOREM 12. If $\mathcal{A}_{n}$ is a star sign pattern of order $n$ with each diagonal entry negative and all non-zero off-diagonal entries positive, then $\mathcal{A}_{n}$ requires $\mathbb{S}_{n}$.

Proof. Without loss of generality, $A \in \mathcal{Q}\left(\mathcal{A}_{n}\right)$ is equivalent to

$$
A=\left[\begin{array}{cccc}
-a_{11} & a_{12} & \ldots & a_{1 n} \\
a_{12} & -a_{22} & 0 & 0 \\
\vdots & 0 & \ddots & \vdots \\
a_{1 n} & 0 & \ldots & -a_{n n}
\end{array}\right]
$$

with specified $a_{i j}>0$. The $(n-1) \times(n-1)$ principal submatrix on rows and columns $\alpha=\{2, \ldots, n\}$ is nonsingular and has $n-1$ negative eigenvalues. Thus, by Haynsworth's theorem [11], every such $A$ has at least $n-1$ negative eigenvalues. The Schur complement formula gives $\operatorname{det}(A)=\operatorname{det}(A[\alpha]) \operatorname{det}(A / A[\alpha])$, where $\operatorname{det}(A / A[\alpha])=-a_{11}+\sum_{k=2}^{n} \frac{a_{1 k}^{2}}{a_{k k}}$. This can be positive, negative, or zero for different choices of $a_{i j}$, implying that $A$ can have only the inertias $(0, n, 0),(0, n-1,1)$, and $(1, n-1,0)$. Thus, $\mathcal{A}_{n}$ requires $\mathbb{S}_{n}$.

6. Concluding remarks. From our results in Section 3, we have proved the statement that all irreducible potentially stable sign patterns of orders 2 and 3 are either sign stable, allow $\mathbb{S}_{3}$, or allow $\mathbb{H}_{3}$. The converse of this statement is clearly true. In Section 4, we have proven the corresponding statement for all tree sign patterns of order 4 (and the superpatterns of those that allow $\mathbb{S}_{4}$ or $\mathbb{H}_{4}$ ). The case of potentially stable non-tree sign patterns of order 4 remains open, as these sign patterns have not been characterized.

The corresponding statement is not true for $n \geq 5$ (even for tree sign patterns), since, for example, with $n=5$ the path sign pattern $00-00 /----$ is sign semi-stable (i.e., the eigenvalues of all realizations have non-positive real parts). It is shown in Proposition 4.2 of [9] that realizations of this sign pattern may only have refined inertia $(0,5,0,0)$ or $(0,3,0,2)$. For $n \geq 6$, a family of sign patterns associated with spider digraphs is sign semi-stable, thus potentially stable, but does not allow $\mathbb{S}_{n}$ or $\mathbb{H}_{n}$; see [2, Section 5]. From our results, it is impossible for a sign pattern of order $n \in\{2,3\}$ and a tree sign pattern of order $n=4$ to have refined inertia $\{(0, n, 0,0),(0, n-2,0,2)\}$ or inertia $\{(0, n, 0),(0, n-1,1)\}$. If, in the statement, sign 
stable is replaced by sign semi-stable, then the converse is false as shown by a skew-symmetric tree sign pattern.

Acknowledgements. The research of PvdD was partially supported by an NSERC Discovery Grant, and AHB wishes to thank St. Olaf College for generously supporting this research. We thank the two anonymous reviewers for several good suggestions that improved this paper, and Xavier Martínez-Rivera for bringing Haynsworth's theorem to our attention.

\section{REFERENCES}

[1] A.H. Berliner, D. DeBlieck, and D. Shah. Sign pattern matrices that allow inertia $\mathbb{S}_{n}$. Involve, 12(7):1229-1240, 2019.

[2] A.H. Berliner, D.D. Olesky, and P. van den Driessche. Sets of refined inertias of zero-nonzero patterns. Linear Algebra and its Applications, 516:243-263, 2017.

[3] A.H. Berliner, D.D. Olesky, P. van den Driessche. Inertia sets allowed by matrix patterns. Electronic Journal of Linear Algebra, 34:343-355, 2018.

[4] E. Bodine, L. Deaett, J.J. McDonald, D.D. Olesky, and P. van den Driessche. Sign patterns that require or allow particular refined inertias. Linear Algebra and its Applications, 437:2228-2242, 2012.

[5] W. Gao, Z. Li, and L. Zhang. Sign patterns that require $\mathbb{H}_{n}$ exist for each $n \geq 4$. Linear Algebra and its Applications, 489:15-23, 2016.

[6] W. Gao, Z. Li, and L. Zhang. Characterization of star sign patterns that require $\mathbb{H}_{n}$. Linear Algebra and its Applications, 499:43-65, 2016.

[7] W. Gao and Z. Li. Path sign patterns of order $n \geq 5$ do not require $\mathbb{H}_{n}$. Linear Algebra and its Applications, 532:99-126, 2017.

[8] Y. Gao and J. Li. On the potential stability of star sign pattern matrices. Linear Algebra and its Applications, 327:61-68, 2001.

[9] C. Garnett, D.D. Olesky, and P. van den Driessche. Refined inertias of tree sign patterns. Electronic Journal of Linear Algebra, 26:620-635, 2013.

[10] C. Garnett, D.D. Olesky, and P. van den Driessche. A note on sign patterns of order 3 that require particular refined inertias. Linear Algebra and its Applications, 450:293-300, 2014.

[11] E. Haynsworth. Determination of the inertia of a partitioned Hermitian matrix. Linear Algebra and its Applications, 1:73-81, 1968.

[12] C.R. Johnson and T.A. Summers. The potentially stable tree sign patterns for dimensions less than five. Linear Algebra and its Applications, 126:1-13, 1989.

[13] I.J. Kim, D.D. Olesky, B.L. Shader, P. van den Driessche, H. van der Holst, and K.N. Vander Meulen. Generating potentially nilpotent full sign patterns. Electronic Journal of Linear Algebra, 18:162-175, 2009.

[14] Q. Lin, D.D. Olesky, and P. van den Driessche. The distance of potentially stable sign patterns to the unstable matrices. SIAM Journal on Matrix Analysis and Applications, 24:356-367, 2002.

[15] J. Maybee and J. Quirk. Qualitative problems in matrix theory. SIAM Review, 11:30-51, 1969.

[16] J. Miyamichi. Sign structures of $3 \times 3$ stable matrices and their generalization to higher-order matrices. Electronics and Communications in Japan, 71:63-73, 1988.

[17] D.D. Olesky, M.F. Rempel, and P. van den Driessche. Refined inertias of tree sign patterns of orders 2 and 3. Involve, 6:1-12, 2013.

[18] P. van den Driessche. Sign Pattern Matrices (Chapter 2). In: R.A. Brualdi, A. Carmona, S. Kirkland, D. Stefanović, and P. van den Driessche, Combinatorial Matrix Theory, Birkhäuser/Springer, Cham, 2018. 